Supporting Information

\title{
Structure and Properties of Poly-a-olefins Containing Quaternary Carbon Centers
}

Ruida Zhao $\neq$, Puke Mi, $\neq$, Sheng $X u^{\sharp}$ and Suqin Dong $\neq$

‡Shanghai Key Laboratory of Advanced Polymeric Materials, Key Laboratory for Ultrafine Materials of Ministry of Education, School of Materials Science and Engineering, East China University of Science and Technology, Shanghai 200237, China

\#School of Chemical and Molecular Engineering, East China University of Science and Technology, Shanghai 200237, China 
Figure S1. ${ }^{1} \mathrm{H}$ NMR spectrum of $\mathrm{C} 20$.

\section{Page S3}

Figure S2. GC chromatograms of 1-Octene PAOs and 1-Hexene PAOs

S4

Table S1. Change in properties of PAO after oxidation

Page

S5 


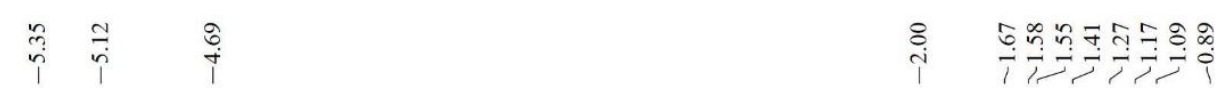

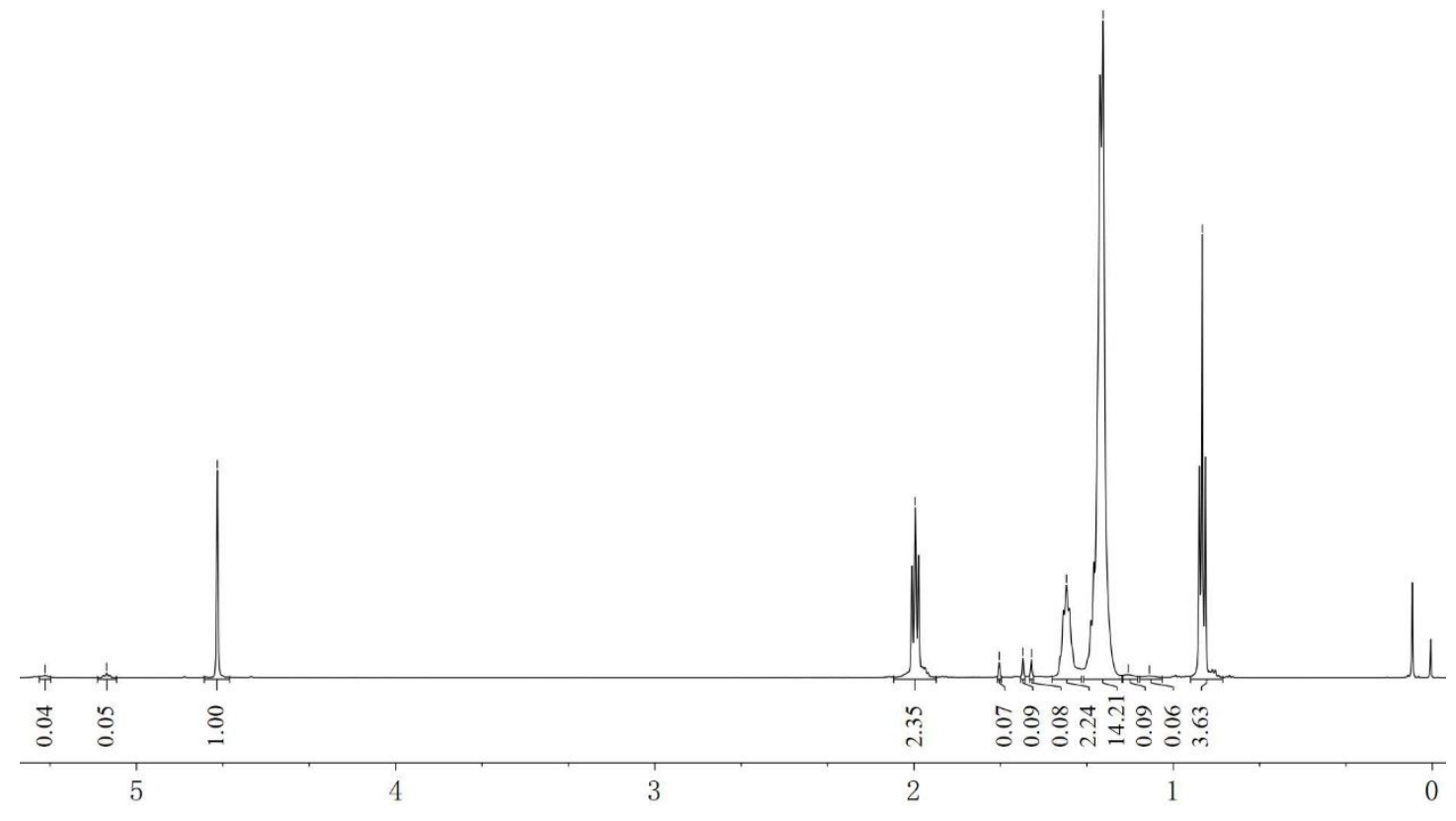

Figure S1. ${ }^{1} \mathrm{H}$ NMR spectrum of C20. 


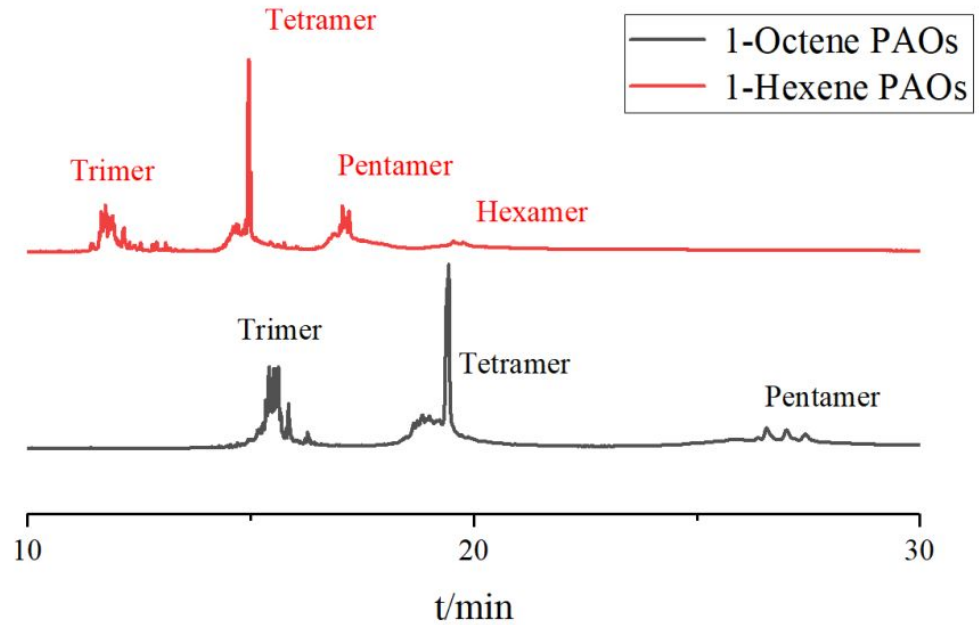

Figure S2. GC chromatograms of 1-Octene PAOs and 1-Hexene PAOs (Run 1,2

Table 4) 
Table S1. Change in properties of PAO after oxidation

\begin{tabular}{cccccccccc}
\hline & \multicolumn{3}{c}{ Kinematic viscosity cSt at $40^{\circ} \mathrm{C}$} & \multicolumn{5}{c}{ Viscosity Index } \\
\cline { 2 - 8 } Base Oil & $\begin{array}{c}\text { Before } \\
\text { Oxidation }\end{array}$ & $24 \mathrm{hr}$ & $48 \mathrm{hr}$ & $72 \mathrm{hr}$ & $\begin{array}{r}\text { Before } \\
\text { Oxidation }\end{array}$ & 24 & 48 & 72 \\
& & & & $\mathrm{hr}$ & $\mathrm{hr}$ \\
\hline PAO without \\
$\begin{array}{c}\text { quaternary carbon } \\
\text { centers }\end{array}$
\end{tabular}

a: $\mathrm{TiCl}_{4}=2.5 \% \mathrm{wt}, \mathrm{V}$ (solvent) $\mathrm{V}(1-$ decene $)=1: 4, \mathrm{nAl} / \mathrm{nTi}=0.5$, reaction temperature $80^{\circ} \mathrm{C}$, reaction time $4 \mathrm{~h}$

b: $\mathrm{TiCl}_{4}=4 \% \mathrm{wt}, \mathrm{V}$ (solvent): $\mathrm{V}(1-$ decene $)=1: 4, \mathrm{nAl} / \mathrm{nTi}=0.5$, reaction temperature $60^{\circ} \mathrm{C}$, reaction time $4 \mathrm{~h} \mathrm{~m}$ (dimer):m(monomer)=1:3

c: Commercial PAO10 base oil from petrolchemical enterprise

Oxidation stability test: weigh 25 g PAO sample into the oxidation vessel, heat up to $100^{\circ} \mathrm{C}$ in oil bath, control the oxygen's flow rate at approximately $10 \mathrm{~L} / \mathrm{h}$, take the sample out and test its viscosity at $40^{\circ} \mathrm{C}$ after certain time (24 hr, $48 \mathrm{hr}, 72 \mathrm{hr}$ ). In addition, viscosity at $100^{\circ} \mathrm{C}$ is also tested and calculate the viscosity index. The data obtained is shown in Table S1. Two kinds of laboratory-made PAO (containing quaternary carbon/typical PAO) and commercial PAO10 base oil products bought from the market were tested. Laboratory-made PAOs were first hydrotreated before oxidation stability test. 